\title{
GLOBAL WEIGHTED ESTIMATES FOR NONLINEAR ELLIPTIC OBSTACLE PROBLEMS OVER REIFENBERG DOMAINS
}

\author{
SUN-SIG BYUN, YUMI CHO, AND DIAN K. PALAGACHEV
}

(Communicated by Tatiana Toro)

\begin{abstract}
We study the obstacle problem for an elliptic equation with discontinuous nonlinearity over a nonsmooth domain, assuming that the irregular obstacle and the nonhomogeneous term belong to suitable weighted Sobolev and Lebesgue spaces, respectively, with weights taken in the Muckenhoupt classes. We establish a Calderón-Zygmund type result by proving that the gradient of the weak solution to the nonlinear obstacle problem has the same weighted integrability as both the gradient of the obstacle and the nonhomogeneous term, provided that the nonlinearity has a small BMO-semi norm with respect to the gradient, and the boundary of the domain is $\delta$-Reifenberg flat. We also get global regularity in the settings of the Morrey and Hölder spaces for the weak solutions to the problem considered.
\end{abstract}

\section{INTRODUCTION}

The general theory of the variational inequalities and free boundary problems is strictly linked to obstacle problems for partial differential equations. These arise naturally in the classical elasticity theory as the simplest unilateral problems in the study of mechanics of elastic membranes and plates when the equilibrium position is sought for an elastic membrane, the boundary of which is keeping fixed and which is constrained to stay above a given obstacle. More generally, the obstacle problems provide a basic tool for the study of variational inequalities and free boundary problems for PDEs and are involved in numerous geometric and potential theory problems such as capacities of sets or minimal surfaces. Their applications have a broad spectrum of problems of modern technology, among them the study of fluid filtration in porous media, melting and crystallization, constrained heating, elasto-plasticity, optimal control problems in the theory of Brownian motion, phase transitions, groundwater hydrology, tumor growth, American type contracts, etc.

Received by the editors August 6, 2013 and, in revised form, January 15, 2014.

2010 Mathematics Subject Classification. Primary 35J87; Secondary 35R05, 35J60, 35B65, $46 \mathrm{E} 35$.

Key words and phrases. Nonlinear elliptic equation, Obstacle problem, Irregular obstacle, Calderón-Zygmund estimate, Muckenhoupt weight, $p$-Laplacean, BMO, Reifenberg flat domain, Morrey space.

The first author was supported by KOSEF-R01-2008-000-11553-0.

The third author is a member of INdAM-GNAMPA. 
We refer the reader to the classical texts by Kinderlehrer and Stampacchia [17, Friedman [15, Rodrigues [26] and Caffarelli 9] for further discussions and more details.

The main goal of the present article is to establish an optimal estimate of Calderón-Zygmund type for the solutions of nonlinear elliptic variational inequalities with obstacle constraints in the settings of weighted Sobolev and Lebesgue spaces.

Throughout the paper we let $p \in(1, \infty)$ to be a fixed real number and $\Omega \subset \mathbb{R}^{n}$ a bounded domain with $n \geq 2$. Given a vector field $\mathbf{a}(\xi, x): \mathbb{R}^{n} \times \mathbb{R}^{n} \rightarrow \mathbb{R}^{n}$, we will suppose it defines a $C^{1}$-Carathéodory map, that is, $\mathbf{a}(\xi, x)$ is differentiable with respect to $\xi$ for almost all (a.a.) $x \in \mathbb{R}^{n}$ and is measurable in $x$ for all $\xi \in \mathbb{R}^{n}$. Moreover, we will assume that there exist constants $0<\mu \leq 1 \leq \Lambda$ such that $\mathbf{a}(\xi, x)$ satisfies the following growth and ellipticity conditions

$$
|\mathbf{a}(\xi, x)|+|\xi|\left|D_{\xi} \mathbf{a}(\xi, x)\right| \leq \Lambda|\xi|^{p-1}
$$

and

$$
D_{\xi} \mathbf{a}(\xi, x) \eta \cdot \eta \geq \mu|\xi|^{p-2}|\eta|^{2}
$$

for a.a. $x \in \mathbb{R}^{n}$ and for all $\xi, \eta \in \mathbb{R}^{n}$.

Given an obstacle $\psi \in W^{1, p}(\Omega)$ such that $\psi \leq 0$ a.e. on $\partial \Omega$, we define the convex admissible set

$$
\mathcal{A}=\left\{v \in W_{0}^{1, p}(\Omega): \quad v \geq \psi \text { a.e. in } \Omega\right\} .
$$

We will deal with a function $u: \Omega \rightarrow \mathbb{R}$, belonging to $\mathcal{A}$, and such that

$$
\int_{\Omega} \mathbf{a}(D u, x) \cdot D(v-u) d x \geq \int_{\Omega}|\mathbf{F}|^{p-2} \mathbf{F} \cdot D(v-u) d x \quad \text { for all } v \in \mathcal{A},
$$

where the nonhomogeneous term $\mathbf{F}$ is a vector valued function in $L^{p}\left(\Omega ; \mathbb{R}^{n}\right)$. According to the common terminology, the function $u$ will be called a solution of the variational inequality (1.4).

It is well known (see [1, 6] for instance) that, under the structure conditions (1.1) and (1.2), there exists a unique solution $u \in \mathcal{A}$ of (1.4). Moreover,

$$
\|D u\|_{L^{p}\left(\Omega ; \mathbb{R}^{n}\right)} \leq c\left(\|\mathbf{F}\|_{L^{p}\left(\Omega ; \mathbb{R}^{n}\right)}+\|D \psi\|_{L^{p}\left(\Omega ; \mathbb{R}^{n}\right)}\right)
$$

with constant $c$ depending only on $n, p, \Lambda$ and $\mu$.

The main result we are going to derive here is a weighted version of the CalderónZygmund regularity estimate. Precisely, assuming $\mathbf{F} \in L_{w}^{p q}\left(\Omega ; \mathbb{R}^{n}\right)$ and $D \psi \in$ $L_{w}^{p q}\left(\Omega ; \mathbb{R}^{n}\right)$, we are interested in a bound of the type

$$
\|D u\|_{L_{w}^{p q}\left(\Omega ; \mathbb{R}^{n}\right)} \leq c\left(\|\mathbf{F}\|_{L_{w}^{p q}\left(\Omega ; \mathbb{R}^{n}\right)}+\|D \psi\|_{L_{w}^{p q}\left(\Omega ; \mathbb{R}^{n}\right)}\right)
$$

holding for each $q \in(1, \infty)$, which in turn implies $D u \in L_{w}^{p q}\left(\Omega ; \mathbb{R}^{n}\right)$. Here $w=w(x)$ is a weight function belonging to the Muckenhoupt class $A_{q}$ (see the discussions in the next section) and $c$ is a constant depending on $n, p, \Lambda$ and $\mu$ as before, and on $\Omega, q$ and $w$ as well.

Needless to say, the basic assumptions (1.1) and (1.2) are not sufficient to ensure the validity of (1.6). One needs to require suitable smoothness of $\mathbf{a}(\xi, x)$ with respect to $x$, and to impose appropriate geometric requirements on the boundary $\partial \Omega$ even in the absence of obstacles (see [8]) or in the absence of weights (see [6]). Motivated by the recent results obtained in [6, 8, we will assume a sort of $x$-integral continuity of $\mathbf{a}(\xi, x)$ measured in terms of small bounded mean oscillation (BMO), 
and a $\delta$-Reifenberg flatness of $\partial \Omega$ (we refer the reader to Section 2 for the precise definitions). These additional hypotheses have proven to be minimal to impose on the data of (1.4) for the validity of the regularity results here obtained. In that sense, the main goal of this paper is to derive the global weighted $L^{p q}$-estimate (1.6) for the gradient of the weak solution to the variational inequality (1.4) under such small-BMO condition and $\delta$-Reifenberg flatness, when the associated weight lies in a suitable Muckenhoupt class. This way, we generalize the recent results from [8] and [6] to elliptic obstacle problems studied in the settings of the weighted Lebesgue spaces.

Various interesting results appeared in the last years which regard nonlinear elliptic problems with irregular obstacles. In [2, Bögelein and Scheven established the property of higher integrability in parabolic variational inequalities with an obstacle constraint. In [1, Bögelein, Duzaar and Mingione derived a local CalderónZygmund estimate for the solution of the variational inequality (1.4) in the case of $\mathbf{a}=\mathbf{a}(\xi)$. Their results were extended in [6] where a global estimate has been proved for nonlinearities $\mathbf{a}=\mathbf{a}(\xi, x)$ under the small-BMO assumption with respect to the $x$-variables and Reifenberg flatness of $\partial \Omega$. We should mention also the paper [13] by Eleuteri and Habermann who considered a quasi-convex functional for a class of obstacle problems with nonstandard growths and established local CalderónZygmund type estimate when the associated integrand is continuous with respect to the $x$-variables. In [27], Scheven showed the existence of localizable solutions and Calderón-Zygmund estimates to parabolic obstacle problems. In [28, Scheven obtained gradient pointwise estimates for a nonlinear elliptic obstacle problem with nonhomogeneous measure data.

Being in the more general framework of the weighted Lebesgue spaces, our results not only complement the works cited above and provide contributions to the general theory of the variational inequalities, but ensure also the possibility to earn finer regularity of solution through special choice of the weight function. In particular, we derive global regularity of the weak solution to (1.4) in the settings of the Morrey spaces which leads to a Hölder continuity result for suitable values of the integrability exponents.

\section{Hypotheses, Preliminaries and Main Results}

In what follows, given a point $y \in \mathbb{R}^{n}$ and a number $\rho>0$, we set $B_{\rho}(y)=\{x \in$ $\left.\mathbb{R}^{n}:|x-y|<\rho\right\}$ for the open ball centered at $y$ and of radius $\rho$.

The main geometric assumption on the boundary of the underlying domain $\Omega$ is its $\delta$-Reifenberg flatness.

Definition 2.1. We say that $\Omega$ is $(\delta, R)$-Reifenberg flat if there exist positive constants $\delta$ and $R$ such that for each $x \in \partial \Omega$ and each $\rho \in(0, R]$ there is a coordinate system $\left\{y_{1}, \cdots, y_{n}\right\}$, which may depend on $x$ and $\rho$, with origin at $x$ and such that

$$
B_{\rho}(x) \cap\left\{y: y_{n}>\delta \rho\right\} \subset B_{\rho}(x) \cap \Omega \subset B_{\rho}(x) \cap\left\{y: y_{n}>-\delta \rho\right\} .
$$

Indeed, the definition is significant for small values of $\delta$, and $R$ could be any constant greater than 1 as it follows by the scaling invariance property. The flatness of the boundary in Reifenberg sense means that it is well approximated by hyperplanes at every point and at each scale. It was Reifenberg who first defined that concept in his studies 25] on Plateau problems, proving that such a domain 
is locally a topological disc for small enough $\delta$. The $\delta$-Reifenberg flatness exhibits a sort of a minimal geometric condition on the boundary ensuring validity of some natural properties of geometric analysis and partial differential equations such as $W^{1, p}$-extension, nontangential accessibility property, measure density condition, the Poincaré inequality and so on. We refer the reader to [12, 19, 20, 31] and the references therein for further details.

It is worth noting that the $C^{1}$-smooth domains are Reifenberg flat with vanishing $\delta$ when $R \searrow 0^{+}$. More generally, Reifenberg flat is any domain with boundary which is locally a graph of Lipschitz continuous function with a small Lipschitz constant. Actually, the class of Reifenberg flat domains goes beyond these common examples and contains domains with rough fractal boundaries. For instance, the von Koch snowflake is a Reifenberg flat when the angle of the spike with respect to the horizontal is small enough.

A remarkable feature of the Reifenberg flat domains, which is an immediate consequence of the definition, is a two-sided variant of the so-called (A)-condition of Ladyzhenskaya and Ural'tseva (cf. [18). Namely, the Lebesgue measure of $B_{\rho}(x) \cap \Omega$ is comparable to the measure of the ball $B_{\rho}(x)$ itself for any $x \in \bar{\Omega}$ and any $\rho \in(0, \operatorname{diam} \Omega)$. In other words, for each $\delta$ there exists a constant $A_{\Omega}(\delta) \in(0,1)$ such that

$$
A_{\Omega}(\delta) \rho^{n} \leq\left|B_{\rho}(x) \cap \Omega\right| \leq\left(1-A_{\Omega}(\delta)\right) \rho^{n} \quad \forall x \in \bar{\Omega}, \forall \rho \in(0, \operatorname{diam} \Omega) .
$$

The lower bound here excludes interior cusps at each point of $\partial \Omega$ and this guarantees the validity of the Sobolev embedding theorem in the spaces $W^{1, p}(\Omega)$. The upper bound in (2.1) instead ensures that no exterior cusps exist on $\partial \Omega$ and this serves to get fine regularity properties of solutions to nonlinear PDEs such as better integrability of the gradient based on reverse Hölder inequalities ( 23, 24] $)$, regularity in Morrey and Hölder spaces of solutions to semilinear problems ([3]) and essential boundedness of the weak solutions to a very general class of quasilinear elliptic equations ([4]). We refer to [12,16,31] for an exhaustive discussion on the properties and regularity of the Reifenberg domains.

To introduce the main assumption regarding the principal part $\mathbf{a}(\xi, x)$ of the nonlinear differential operator considered, we define

$$
\Theta\left(\mathbf{a}, B_{\rho}(y)\right)(x)=\sup _{\xi \in \mathbb{R}^{n} \backslash\{0\}} \frac{\left|\mathbf{a}(\xi, x)-\overline{\mathbf{a}}_{B_{\rho}(y)}(\xi)\right|}{|\xi|^{p-1}},
$$

where $\overline{\mathbf{a}}_{B_{\rho}(y)}(\xi)$ stands for the integral average

$$
f_{B_{\rho}(y)} \mathbf{a}(\xi, z) d z=\frac{1}{\left|B_{\rho}(y)\right|} \int_{B_{\rho}(y)} \mathbf{a}(\xi, z) d z
$$

of $\mathbf{a}(\xi, \cdot)$ over $B_{\rho}(y)$.

Definition 2.2. We say that $\mathbf{a}(\xi, x)$ is $(\delta, R)$-vanishing if

$$
\sup _{0<\rho \leq R} \sup _{y \in \mathbb{R}^{n}} f_{B_{\rho}(y)}\left|\Theta\left(\mathbf{a}, B_{\rho}(y)\right)(x)\right| d x \leq \delta .
$$

It is worth noting that the $(\delta, R)$-vanishing of $\mathbf{a}(\xi, x)$ means that the function $x \mapsto \frac{\mathbf{a}(\xi, x)}{|\xi|^{p-1}}$ has a small mean oscillation around its integral average, uniformly in $\xi$. This allows, of course, discontinuities of $\mathbf{a}(\xi, x)$ with respect to $x$, measured in terms of smallness of the BMO-seminorm. That is a quite general condition to 
impose on the behaviour of $\mathbf{a}(\xi, x)$ in $x$ which is minimal in some sense, and which is surely satisfied if $x \mapsto \frac{\mathbf{a}(\xi, x)}{|\xi|^{p-1}}$ is VMO or continuous with respect to $x$, uniformly in $\xi$ (see $6,8,21$, and the references therein).

For what concerns the constant $R$ in Definitions 2.1 and 2.2 , it could be any positive number due to the scaling invariance property of the problem (1.4), while $\delta$ remains the same under such a scaling. Further on, the constant $\delta$ will be sufficiently small to be selected in a universal way so that it will be independent of the nonhomogeneous term $\mathbf{F}$ and the obstacle $\psi$.

For the purposes of this paper, $\mathbf{F}$ and $D \psi$ will be taken to lie in an appropriate weighted Lebesgue space. For the sake of completeness, let us recall the definition of the Muckenhoupt classes $A_{q}$ of weights with $1<q<\infty$. A weight $w$ is a positive, locally integrable function on $\mathbb{R}^{n}$. Given $q \in(1, \infty)$, the class $A_{q}$ is defined as the collection of all weights $w$ for which

$$
[w]_{q}=\sup _{y \in \mathbb{R}^{n}} \sup _{r>0}\left(f_{B_{r}(y)} w(x) d x\right)\left(f_{B_{r}(y)} w(x)^{\frac{-1}{q-1}} d x\right)^{q-1}<\infty .
$$

A typical example of a weight in $A_{q}$ is given by the function $w_{\sigma}(x)=|x|^{\sigma}$ when $-n<\sigma<n(q-1)$.

Now, the weighted Lebesgue space $L_{w}^{q}(\Omega)$ related to $A_{q}$ consists of all measurable functions $f: \Omega \rightarrow \mathbb{R}^{n}$ such that

$$
\|f\|_{L_{w}^{q}(\Omega)}=\left(\int_{\Omega}|f(x)|^{q} w(x) d x\right)^{\frac{1}{q}}<\infty .
$$

We set further $w(E)$ for the weighted Lebesgue measure of a measurable set $E \subset \mathbb{R}^{n}$, given by

$$
w(E)=\int_{E} w(x) d x .
$$

In the sequel, we will use the following relationship between the Lebesgue and the weighted measures.

Lemma 2.3 (see [21]). Let $E$ a measurable subset of $\Omega$ and $w \in A_{q}$ for some $1<q<\infty$.

Then there exist positive constants $\nu$ and $\alpha$, depending only on $[w]_{q}$ and $n$, such that

$$
\frac{1}{\alpha}\left(\frac{\left|E \cap B_{r}(y)\right|}{\left|B_{r}(y)\right|}\right)^{q} \leq \frac{w\left(E \cap B_{r}(y)\right)}{w\left(B_{r}(y)\right)} \leq \alpha\left(\frac{\left|E \cap B_{r}(y)\right|}{\left|B_{r}(y)\right|}\right)^{\nu} .
$$

The main result of the paper is the next theorem.

Theorem 2.4. Let $1<p<\infty$ and $w$ be a weight in $A_{q}$ for some $q \in(1, \infty)$. Suppose that $|\mathbf{F}|^{p} \in L_{w}^{q}(\Omega)$ and $|D \psi|^{p} \in L_{w}^{q}(\Omega)$.

There exists a positive constant $\delta=\delta\left(n, p, q, \Lambda, \mu,[w]_{q}\right)$ such that if $\mathbf{a}(\xi, x)$ is $(\delta, R)$-vanishing and $\Omega$ is $(\delta, R)$-Reifenberg flat, then the gradient $D u$ of the weak solution to the variational inequality (1.4) satisfies $|D u|^{p} \in L_{w}^{q}(\Omega)$ and we have the estimate

$$
\int_{\Omega}|D u|^{p q} w(x) d x \leq c\left(\int_{\Omega}|\mathbf{F}|^{p q} w(x) d x+\int_{\Omega}|D \psi|^{p q} w(x) d x\right)
$$

with a constant $c$ depending only on $n, p, q, \Lambda, \mu,[w]_{q}$ and $\Omega$. 
Let us point out to the reader's attention the fact that in the special, unweighted case $(w=1)$ Theorem 2.4 gives rise to a regularity result already proved in the earlier paper [6]. The technique employed in proving (2.5) is based on local comparison estimates, maximal function and Vitali covering lemma, and is more or less analogous to that in 6, adapted to the settings of the weighted spaces here considered. Indeed, that is possible thanks to Lemma 2.3 which implies the associated weight measure is comparable to the Lebesgue one. In that sense, Theorem 2.4 is a natural extension of the work in [6] to the framework of weighted Lebesgue spaces.

To proceed further with our second result, which is a particular outgrowth of Theorem 2.4 we need to recall the definition of the Morrey classes. Namely, given $p \in(1, \infty)$ and $\gamma \in(0, n)$, the Morrey space $L^{p, \gamma}(\Omega)$ is the collection of all functions $f \in L^{p}(\Omega)$ for which

$$
\|f\|_{L^{p, \gamma}(\Omega)}=\sup _{\substack{x_{0} \in \Omega \\ r \in(0, \text { diam } \Omega)}}\left(\frac{1}{r^{\gamma}} \int_{B_{r}\left(x_{0}\right) \cap \Omega}|f(x)|^{p} d x\right)^{1 / p}<\infty .
$$

That quantity defines a norm which makes $L^{p, \gamma}(\Omega)$ a Banach space. The limit cases $\gamma=0$ and $\gamma=n$ give rise to $L^{p}(\Omega)$ and $L^{\infty}(\Omega)$, respectively.

Our second result yields Sobolev-Morrey regularity of the weak solution to the variational inequality (1.4) and follows from Theorem 2.4 with a particular choice of the weight.

Theorem 2.5. Given $p, q \in(1, \infty)$ and $\gamma \in(0, n)$, assume that $|\mathbf{F}|^{p} \in L^{q, \gamma}(\Omega)$ and $|D \psi|^{p} \in L^{q, \gamma}(\Omega)$.

There exists a positive constant $\delta=\delta(n, p, q, \gamma, \Lambda, \mu)$ such that if $\mathbf{a}(\xi, x)$ is $(\delta, R)$ vanishing and $\Omega$ is $(\delta, R)$-Reifenberg flat, then the gradient $D u$ of the weak solution to the variational inequality (1.4) belongs to the Morrey space $L^{p q, \gamma}\left(\Omega ; \mathbb{R}^{n}\right)$ and satisfies the estimate

$$
\|D u\|_{L^{p q, \gamma}\left(\Omega ; \mathbb{R}^{n}\right)} \leq c\left(\|\mathbf{F}\|_{L^{p q, \gamma}\left(\Omega ; \mathbb{R}^{n}\right)}+\|D \psi\|_{L^{p q, \gamma}\left(\Omega ; \mathbb{R}^{n}\right)}\right)
$$

with a constant $c$ depending only on $n, p, q, \gamma, \Lambda, \mu$ and $\Omega$.

An important consequence of Theorem 2.5. based on the known properties of functions having gradients in Morrey spaces (cf. [10]) and the (A)-condition (2.1) of $\partial \Omega$, is the next Corollary which asserts better integrability and even Hölder continuity of the weak solution to the variational inequality (1.4) for appropriate values of $p, q$ and $\gamma$.

Corollary 2.6. Under the hypotheses of Theorem 2.5, let $u \in W_{0}^{1, p}(\Omega)$ be a weak solution to the variational inequality (1.4).

Then

(1) $u \in L^{\frac{n p q}{n-p q}, \frac{n \gamma}{n-p q}}(\Omega) \subset L^{p q, \gamma+p q}(\Omega)$ if $p q+\gamma<n$;

(2) $u \in L^{\tilde{p}, \tilde{\gamma}}(\Omega)$ for any $\tilde{p}<\infty$ and any $\tilde{\gamma}<n$, if $p q+\gamma=n$;

(3) $u \in C^{0,1-\frac{n-\gamma}{p q}}(\bar{\Omega})$ if $p q+\gamma>n$.

Let us point out that, without essential difficulties, the result of Theorem 2.5 could be extended to the case of variational inequalities in the settings of the generalized Morrey spaces (cf. 29]). 


\section{Proofs of the Main Results}

We start this section with reviewing some standard properties of the maximal function, and basic facts from the measure theory with respect to the Muckenhoupt weights.

Our approach in proving Theorem 2.4 is based on the Hardy-Littlewood maximal function operator. Recall that the maximal function $\mathcal{M} h$ of a locally integrable function $h$ is given by

$$
(\mathcal{M} h)(x)=\sup _{\rho>0} f_{B_{\rho}(x)}|h(y)| d y .
$$

Indeed, if $h$ is defined only on a bounded domain $U$, then we assume tacitly that it is extended as zero outside $U$ and then apply the maximal operator.

It follows from [21,22, 30 that if a weight $w$ belongs to the Muckenhoupt class $A_{q}$ for some $q \in(1, \infty)$, then there exists a constant $c=c\left(n, q,[w]_{q}\right)>0$ such that

$$
\frac{1}{c}\|h\|_{L_{w}^{q}\left(\mathbb{R}^{n}\right)} \leq\|\mathcal{M} h\|_{L_{w}^{q}\left(\mathbb{R}^{n}\right)} \leq c\|h\|_{L_{w}^{q}\left(\mathbb{R}^{n}\right)} \quad \text { for each } h \in L_{w}^{q}\left(\mathbb{R}^{n}\right) .
$$

In the particular case when $w(x) \equiv 1$, we have

$$
\left|\left\{x \in \mathbb{R}^{n}:(\mathcal{M} h)(x)>\lambda\right\}\right| \leq \frac{c}{\lambda} \int|h(x)| d x \text { for every } \lambda>0
$$

with a constant $c=c(n)$.

We will use the following technical lemma, the proof of which can be found in [21, 30].

Lemma 3.1. Assume that $h$ is a nonnegative measurable function on a bounded subset $U$ of $\mathbb{R}^{n}$. Let $\theta>0$ and $m>1$ be constants and $w \in A_{q}$ with $0<q<\infty$.

Then

$$
h \in L_{w}^{q}(U) \Longleftrightarrow S=\sum_{k \geq 1} m^{k q} w\left(\left\{x \in U: h(x)>\theta m^{k}\right\}\right)<\infty
$$

and

$$
\frac{1}{c} S \leq\|h\|_{L_{w}^{q}(U)}^{q} \leq c(w(U)+S),
$$

where $c>0$ is a constant depending only on $\theta, m$ and $q$.

The following Vitali-type covering lemma will be useful in the sequel. We refer the reader to [5, Lemma 3.3] or [21, Lemma 3.8] for the corresponding proof.

Lemma 3.2. Assume that $\Omega$ is a bounded domain satisfying the $(\delta, R)$-Reifenberg flatness condition with $0<\delta<\frac{1}{8}$, and $w \in A_{q}$ for some $q \in(1, \infty)$. Let $C$ and $D$ be measurable sets with $C \subset D \subset \Omega$. Assume further that there exists a small constant $\epsilon>0$ such that

$$
w\left(C \cap B_{1}(y)\right)<\epsilon w\left(B_{1}(y)\right) \quad \forall y \in \Omega
$$

and, for every $y \in \Omega$ and for every $r \in(0,1)$ one has

$$
B_{r}(y) \cap \Omega \subset D \quad \text { whenever } w\left(C \cap B_{r}(y)\right) \geq \epsilon w\left(B_{r}(y)\right) .
$$

Then

$$
w(C) \leq c^{*} \epsilon w(D)
$$

with a positive constant $c^{*}$ depending only on $n, q$ and $[w]_{q}$. 
The next lemma is the main ingredient of the principal result in [6] which treats unweighted variational inequalities.

Lemma 3.3 (see 6, Lemma 4.8]). Assume that $|\mathbf{F}| \in L^{p}(\Omega)$ and $|D \psi| \in L^{p}(\Omega)$ with $\psi \leq 0$ a.e. on $\partial \Omega$. Suppose that $u \in W_{0}^{1, p}(\Omega)$ is a weak solution of the variational inequality (1.4).

Then there exists a universal constant $N=N(\mu, \Lambda, n, p)>1$ so that for every fixed $0<\epsilon<1$ one can find a small enough $\delta=\delta(\epsilon, \mu, \Lambda, n, p)>0$ with the property that if $\mathbf{a}(\xi, x)$ is $(\delta, 42)$-vanishing, $\Omega$ is $(\delta, 42)$-Reifenberg flat, and $B_{r}(y)$, with $y \in \Omega$ and $r \in(0,1)$, satisfies

$$
\left|\left\{x \in \Omega \cap B_{r}(y): \mathcal{M}\left(|D u|^{p}\right)>N^{p}\right\}\right| \geq \epsilon\left|B_{r}(y)\right|,
$$

then we have

$$
\begin{aligned}
B_{r}(y) \cap \Omega \subset\{ & \left\{x \in \Omega: \mathcal{M}\left(|D u|^{p}\right)>1\right\} \\
& \cup\left\{x \in \Omega: \mathcal{M}\left(|\mathbf{F}|^{p}\right)>\delta^{p}\right\} \cup\left\{x \in \Omega: \mathcal{M}\left(|D \psi|^{p}\right)>\delta^{p}\right\} .
\end{aligned}
$$

The weighted counterpart of Lemma 3.3 follows, which relies on Lemma 2.3

Lemma 3.4. Assume that $w \in A_{q}$ for some $q \in(1, \infty),|\mathbf{F}| \in L_{w}^{p}(\Omega)$ and $|D \psi| \in$ $L_{w}^{p}(\Omega)$ with $\psi \leq 0$ a.e. on $\partial \Omega$. Suppose that $u \in W_{0}^{1, p}(\Omega)$ is the weak solution of the variational inequality (1.4).

Then there exists a universal constant $N=N(\mu, \Lambda, n, p)>1$ such that for every fixed $0<\epsilon<1$ one can find small $\delta=\delta\left(\epsilon, \mu, \Lambda, n, p,[w]_{q}\right)>0$ such that if $\mathbf{a}(\xi, x)$ is $(\delta, 42)$-vanishing, $\Omega$ is $(\delta, 42)$-Reifenberg flat, and $B_{r}(y)$ with $y \in \Omega$ and $r \in(0,1)$ satisfies

$$
w\left(\left\{x \in \Omega \cap B_{r}(y): \mathcal{M}\left(|D u|^{p}\right)>N^{p}\right\}\right) \geq \epsilon w\left(B_{r}(y)\right),
$$

then we have

$$
\begin{aligned}
B_{r}(y) \cap \Omega \subset\{ & \left\{x \in \Omega: \mathcal{M}\left(|D u|^{p}\right)>1\right\} \\
& \cup\left\{x \in \Omega: \mathcal{M}\left(|\mathbf{F}|^{p}\right)>\delta^{p}\right\} \cup\left\{x \in \Omega: \mathcal{M}\left(|D \psi|^{p}\right)>\delta^{p}\right\} .
\end{aligned}
$$

Proof. By Lemmas 2.3 and 3.4 we have

$$
\begin{aligned}
\mid\{x \in & \left.\Omega: \mathcal{M}\left(|D u|^{p}\right)>N^{p}\right\} \cap B_{r}(y) \mid \\
& \geq\left(\frac{1}{\alpha} \frac{w\left(\left\{x \in \Omega: \mathcal{M}\left(|D u|^{p}\right)>N^{p}\right\} \cap B_{r}(y)\right)}{w\left(B_{r}(y)\right)}\right)^{\frac{1}{\nu}}\left|B_{r}(y)\right| \\
& \geq\left(\frac{\epsilon}{\alpha}\right)^{1 / \nu}\left|B_{r}(y)\right|
\end{aligned}
$$

according to (3.7). We apply now Lemma 3.4 with $\epsilon$ replaced by $\left(\frac{\epsilon}{\alpha}\right)^{\frac{1}{\nu}}$ and select $\delta=\delta\left(\epsilon, \mu, \Lambda, n, p,[w]_{q}\right)$ in order to get (3.8).

Proof of Theorem 2.4. We assert first of all that there exists a universal constant $c$ depending on $n, p, q, \mu, \Lambda, \Omega$ and $[w]_{q}$ such that

$$
\|D u\|_{L_{w}^{p q}(\Omega)} \leq c
$$

if the nonhomogeneous term $\mathbf{F}$ and the obstacle $\psi$ satisfy

$$
\|\mathbf{F}\|_{L_{w}^{p q}\left(\Omega ; \mathbb{R}^{n}\right)}+\|D \psi\|_{L_{w}^{p q}(\Omega)} \leq \delta .
$$


In fact, the Hölder inequality implies

$$
\|\mathbf{F}\|_{L^{p}\left(\Omega ; \mathbb{R}^{n}\right)}^{p} \leq \int_{\Omega}|\mathbf{F}|^{p} w^{\frac{1}{q}} w^{-\frac{1}{q}} d x \leq\left(\int_{\Omega}|\mathbf{F}|^{p q} w d x\right)^{\frac{1}{q}} \underbrace{\left(\int_{\Omega} w^{\frac{-1}{q-1}} d x\right)^{\frac{q-1}{q}}}_{I}
$$

Since $\Omega$ is bounded, we have $\Omega \subset B_{\frac{d}{2}}\left(x_{0}\right)$ for some $x_{0} \in \Omega$ where $d$ stands for the diameter of $\Omega$. Then, employing (2.3) and (2.4), we estimate $I$ as follows

$$
\begin{aligned}
\left(\int_{\Omega} w^{\frac{-1}{q-1}} d x\right)^{q-1} & \leq\left(\int_{B_{\frac{d}{2}}\left(x_{0}\right)} w^{\frac{-1}{q-1}} d x\right)^{q-1} \\
& \leq \frac{\left(f_{B_{\frac{d}{2}}\left(x_{0}\right)} w d x\right)\left(f_{B_{\frac{d}{2}}\left(x_{0}\right)} w^{\frac{-1}{q-1}} d x\right)^{q-1}\left|B_{\frac{d}{2}}\left(x_{0}\right)\right|^{q-1}}{f_{B_{\frac{d}{2}}\left(x_{0}\right)} w d x} \\
& \leq \frac{\left|B_{\frac{d}{2}}\left(x_{0}\right)\right|^{q}}{w\left(B_{\frac{d}{2}}\left(x_{0}\right)\right)}[w]_{q} \\
& \leq \frac{d^{n q}\left|B_{1}\right|^{q}}{w(\Omega)}[w]_{q} .
\end{aligned}
$$

This estimate, combined with (3.10) and (3.11), gives

$$
\|\mathbf{F}\|_{L^{p}\left(\Omega ; \mathbb{R}^{n}\right)}^{p} \leq \frac{d^{n}\left|B_{1}\right|}{w(\Omega)^{\frac{1}{q}}}[w]_{q}^{\frac{1}{q}} \delta^{p}
$$

In the same manner we get

$$
\|D \psi\|_{L^{p}(\Omega)}^{p} \leq \frac{d^{n}\left|B_{1}\right|}{w(\Omega)^{\frac{1}{q}}}[w]_{q}^{\frac{1}{q}} \delta^{p}
$$

whence

$$
\|\mathbf{F}\|_{L^{p}\left(\Omega ; \mathbb{R}^{n}\right)}^{p}+\|D \psi\|_{L^{p}(\Omega)}^{p} \leq c \delta^{p}
$$

with a suitable constant $c=c\left(n, q,[w]_{q}, \Omega\right)$.

Now take $N$ and $\epsilon$ and select the corresponding $\delta>0$ as given by Lemma 3.4 . Set further

$$
C=\left\{x \in \Omega: \mathcal{M}\left(|D u|^{p}\right)>N^{p}\right\}
$$

and

$$
D=\left\{x: \mathcal{M}\left(|D u|^{p}\right)>1\right\} \cup\left\{x: \mathcal{M}\left(|\mathbf{F}|^{p}\right)>\delta^{p}\right\} \cup\left\{x: \mathcal{M}\left(|D \psi|^{p}\right)>\delta^{p}\right\} .
$$


The estimates (3.2) and (1.5) yield that for any $y \in \Omega$ and for some constant $c=c\left(\mu, \Lambda, n, p, q, \Omega,[w]_{q}\right)$ one has

$$
\begin{aligned}
\left|C \cap B_{1}(y)\right| & \leq c \int_{\Omega \cap B_{1}(y)}|D u|^{p} d x \\
& \leq c\left(\|\mathbf{F}\|_{L^{p}\left(\Omega ; \mathbb{R}^{n}\right)}^{p}+\|D \psi\|_{L^{p}(\Omega)}^{p}\right) \\
& \leq c \delta^{p} \\
& \leq\left(\frac{\epsilon}{\alpha}\right)^{\frac{1}{\nu}}\left|B_{1}(y)\right|,
\end{aligned}
$$

where $\delta$ is additionally taken small enough, if necessary, in order to ensure the last inequality. We then apply Lemma 2.3 in order to get

$$
w\left(C \cap B_{1}(y)\right) \leq \alpha\left(\frac{\left|C \cap B_{1}(y)\right|}{\left|B_{1}(y)\right|}\right)^{\nu} w\left(B_{1}(y)\right) \leq \epsilon w\left(B_{1}(y)\right) .
$$

At this point the hypotheses of Lemma 3.2 hold because of (3.13) and Lemma 3.4. and as consequence we have

$$
\begin{aligned}
& w\left(\left\{x \in \Omega: \mathcal{M}\left(|D u|^{p}\right)>N^{p}\right\}\right) \leq \epsilon_{1} w\left(\left\{x \in \Omega: \mathcal{M}\left(|D u|^{p}\right)>1\right\}\right) \\
&+\epsilon_{1}\left[w\left(\left\{x \in \Omega: \mathcal{M}\left(|\mathbf{F}|^{p}\right)>\delta^{p}\right\}\right)\right. \\
&\left.+w\left(\left\{x \in \Omega: \mathcal{M}\left(|D \psi|^{p}\right)>\delta^{p}\right\}\right)\right]
\end{aligned}
$$

with $\epsilon_{1}=c^{*} \epsilon$ and $c^{*}$ depending only on $n, q$ and $[w]_{q}$.

Running induction in $k$, yields the following power decay estimate:

$$
\begin{aligned}
w\left(\left\{x \in \Omega: \mathcal{M}\left(|D u|^{p}\right)>N^{p k}\right\}\right) \leq & \epsilon_{1}^{k} w\left(\left\{x \in \Omega: \mathcal{M}\left(|D u|^{p}\right)>1\right\}\right) \\
& +\sum_{i=1}^{k} \epsilon_{1}^{i} w\left(\left\{x \in \Omega: \mathcal{M}\left(|\mathbf{F}|^{p}\right)>\delta^{p} N^{p(k-i)}\right\}\right) \\
& +\sum_{i=1}^{k} \epsilon_{1}^{i} w\left(\left\{x \in \Omega: \mathcal{M}\left(|D \psi|^{p}\right)>\delta^{p} N^{p(k-i)}\right\}\right) .
\end{aligned}
$$

Then, using that estimate, we compute as follows

$$
\begin{aligned}
\sum_{k=1}^{\infty} N^{p q k} w(\{x & \left.\left.\in \Omega: \mathcal{M}\left(|D u|^{p}\right)>N^{p k}\right\}\right) \\
\leq & \sum_{k=1}^{\infty}\left(N^{p q} \epsilon_{1}\right)^{k} w\left(\left\{x \in \Omega: \mathcal{M}\left(|D u|^{p}\right)>1\right\}\right) \\
& +\sum_{i=1}^{\infty}\left(N^{p q} \epsilon_{1}\right)^{i} \underbrace{\sum_{k=i}^{\infty} N^{p q(k-i)} w\left(\left\{x \in \Omega: \mathcal{M}\left(|\mathbf{F}|^{p}\right)>\delta^{p} N^{p(k-i)}\right\}\right)}_{S_{1}} \\
& +\sum_{i=1}^{\infty}\left(N^{p q} \epsilon_{1}\right)^{i} \underbrace{\sum_{k=i}^{\infty} N^{p q(k-i)} w\left(\left\{x \in \Omega: \mathcal{M}\left(|D \psi|^{p}\right)>\delta^{p} N^{p(k-i)}\right\}\right)}_{S_{2}} \\
\leq & \sum_{k=1}^{\infty}\left(N^{p q} \epsilon_{1}\right)^{k} w(\Omega)+\sum_{i=1}^{\infty}\left(N^{p q} \epsilon_{1}\right)^{i}\left[S_{1}+S_{2}\right] .
\end{aligned}
$$


In view of Lemma 3.1, (3.1) and (3.10), we have

$$
S_{1} \leq c \frac{1}{\delta}\|\mathbf{F}\|_{L_{w}^{p q}\left(\Omega ; \mathbb{R}^{n}\right)} \leq c
$$

and

$$
S_{2} \leq c \frac{1}{\delta}\|D \psi\|_{L_{w}^{p q}(\Omega)} \leq c
$$

for some constant $c=c\left(n, p, q, \mu, \Lambda,[w]_{q}\right)$.

This way, we conclude that there exists a constant $c=c\left(n, p, q, \mu, \Lambda, \Omega,[w]_{q}\right)$ such that

$$
\sum_{k=1}^{\infty} N^{p q k} w\left(\left\{x \in \Omega: \mathcal{M}\left(|D u|^{p}\right)>N^{p k}\right\}\right) \leq c \sum_{k=1}^{\infty}\left(N^{p q} \epsilon_{1}\right)^{k} \leq c,
$$

after choosing $\epsilon$ so small that $N^{p q} \epsilon_{1}<1$.

According to Lemma 3.4 we find a corresponding $\delta>0$ which depends on $n, p, q, \mu, \Lambda,[w]_{q}$ and $\Omega$, and the claim (3.9) follows from (3.1).

To proceed further, we consider the normalized functions

$$
u_{\lambda}=\frac{u}{\lambda}, \quad \mathbf{F}_{\lambda}=\frac{\mathbf{F}}{\lambda}, \quad \psi_{\lambda}=\frac{\psi}{\lambda},
$$

where $\lambda=\frac{1}{\delta}\left(\|\mathbf{F}\|_{L_{w}^{p q}\left(\Omega ; \mathbb{R}^{n}\right)}+\|D \psi\|_{L_{w}^{p q}(\Omega)}\right)$. It follows that

$$
\left\|\mathbf{F}_{\lambda}\right\|_{L_{w}^{p q}\left(\Omega ; \mathbb{R}^{n}\right)}+\left\|D \psi_{\lambda}\right\|_{L_{w}^{p q}(\Omega)} \leq \delta,
$$

which implies that for some constant $c=c\left(n, p, q, \mu, \Lambda, \Omega,[w]_{q}\right)$, one has

$$
\left\|D u_{\lambda}\right\|_{L_{w}^{p q}(\Omega)} \leq c .
$$

Indeed, the last bound leads to the desired estimate

$$
\|D u\|_{L_{w}^{p q}(\Omega)} \leq c\left(\|\mathbf{F}\|_{L_{w}^{p q}\left(\Omega ; \mathbb{R}^{n}\right)}+\|D \psi\|_{L_{w}^{p q}(\Omega)}\right)
$$

and this completes the proof of Theorem 2.4

Proof of Theorem 2.5. Without loss of generality we may assume that the nonhomogeneous term $\mathbf{F}$ and the gradient of the obstacle $D \psi$ are taken to be zero outside $\Omega$, so that

$$
\|\mathbf{F}\|_{L^{p q, \gamma}\left(\mathbb{R}^{n} ; \mathbb{R}^{n}\right)}=\|\mathbf{F}\|_{L^{p q, \gamma}\left(\Omega ; \mathbb{R}^{n}\right)} \quad \text { and } \quad\|D \psi\|_{L^{p q, \gamma}\left(\mathbb{R}^{n} ; \mathbb{R}^{n}\right)} \leq c\|D \psi\|_{L^{p q, \gamma}\left(\Omega ; \mathbb{R}^{n}\right)} .
$$

Let $x_{0} \in \Omega$ and $r>0$ be arbitrary. We set $\chi_{B_{r}\left(x_{0}\right)}$ for the characteristic function of the ball $B_{r}\left(x_{0}\right)$ and $\mathcal{M} \chi_{B_{r}\left(x_{0}\right)}(x)$ for its Hardy-Littlewood maximal function.

For an arbitrary exponent $\sigma \in(0,1)$, it is a classical fact (see e.g. Proposition 2 in [11]) that

$$
\mathcal{M}\left(\left(\mathcal{M} \chi_{B_{r}\left(x_{0}\right)}(x)\right)^{\sigma}\right) \leq c\left(\mathcal{M} \chi_{B_{r}\left(x_{0}\right)}(x)\right)^{\sigma} \quad \text { for a.a. } x \in \mathbb{R}^{n} .
$$

In other words, $\left(\mathcal{M} \chi_{B_{r}\left(x_{0}\right)}(x)\right)^{\sigma}$ belongs to the Muckenhoupt class $A_{1}$ and therefore $\left(\mathcal{M} \chi_{B_{r}\left(x_{0}\right)}(x)\right)^{\sigma} \in A_{q}$ for each $q \in(1, \infty)$ with

$$
\left[\left(\mathcal{M} \chi_{B_{r}\left(x_{0}\right)}(x)\right)^{\sigma}\right]_{q}=c(n, q, \sigma) .
$$

Fix now an arbitrary $\sigma \in(\gamma / n, 1)$ and apply Theorem 2.4. It follows that there exist constants $\delta>0$ and $c$, depending on $n, p, q, \Lambda, \mu, \sigma$ and $\Omega$, such that if $\mathbf{a}(\xi, x)$ 
is $(\delta, R)$-vanishing and $\Omega$ is $(\delta, R)$-Reifenberg flat, the estimate (2.5) yields

$$
\begin{aligned}
\int_{B_{r}\left(x_{0}\right) \cap \Omega}|D u(x)|^{p q} d x & =\int_{\Omega}|D u(x)|^{p q}\left(\chi_{B_{r}\left(x_{0}\right)}(x)\right)^{\sigma} d x \\
& \leq \int_{\Omega}|D u(x)|^{p q}\left(\mathcal{M} \chi_{B_{r}\left(x_{0}\right)}(x)\right)^{\sigma} d x \\
& \leq c \int_{\Omega}|K(x)|^{p q}\left(\mathcal{M} \chi_{B_{r}\left(x_{0}\right)}(x)\right)^{\sigma} d x \\
& =c \int_{\mathbb{R}^{n}}|K(x)|^{p q}\left(\mathcal{M} \chi_{B_{r}\left(x_{0}\right)}(x)\right)^{\sigma} d x
\end{aligned}
$$

where we have set

$$
K(x)=|\mathbf{F}(x)|+|D \psi(x)|
$$

for the sake of simplicity.

With the aid of the dyadic decomposition

$$
\mathbb{R}^{n}=B_{2 r}\left(x_{0}\right) \bigcup\left(\bigcup_{k=1}^{\infty} B_{2^{k+1} r}\left(x_{0}\right) \backslash B_{2^{k} r}\left(x_{0}\right)\right)
$$

the last integral above decomposes into

$$
\int_{\mathbb{R}^{n}}|K(x)|^{p q}\left(\mathcal{M} \chi_{B_{r}\left(x_{0}\right)}(x)\right)^{\sigma} d x=I_{0}\left(r, x_{0}\right)+\sum_{k=1}^{\infty} I_{k}\left(r, x_{0}\right),
$$

with

$$
\begin{aligned}
& I_{0}\left(r, x_{0}\right)=\int_{B_{2 r}\left(x_{0}\right)}|K(x)|^{p q}\left(\mathcal{M} \chi_{B_{r}\left(x_{0}\right)}(x)\right)^{\sigma} d x, \\
& I_{k}\left(r, x_{0}\right)=\int_{B_{2^{k+1} r_{r}}\left(x_{0}\right) \backslash B_{2^{k_{r}}}\left(x_{0}\right)}|K(x)|^{p q}\left(\mathcal{M} \chi_{B_{r}\left(x_{0}\right)}(x)\right)^{\sigma} d x .
\end{aligned}
$$

We use the inequality $\mathcal{M} \chi_{B_{r}\left(x_{0}\right)}(x) \leq 1$ a.e. $\mathbb{R}^{n}$ in order to estimate $I_{0}\left(r, x_{0}\right)$. Namely,

$$
\begin{aligned}
I_{0}\left(r, x_{0}\right) & \leq \int_{B_{2 r}\left(x_{0}\right)}|K(x)|^{p q} d x \leq c(n) r^{\gamma}\|K\|_{L^{p q, \gamma}(\Omega)}^{p q} \\
& \leq c(n) r^{\gamma}\left(\|\mathbf{F}\|_{L^{p q, \gamma}\left(\Omega ; \mathbb{R}^{n}\right)}^{p q}+\|D \psi\|_{L^{p q, \gamma}\left(\Omega ; \mathbb{R}^{n}\right)}^{p q}\right) .
\end{aligned}
$$

Later on, it is clear that

$$
\frac{1}{\left|B_{\rho}(x)\right|} \int_{B_{\rho}(x)}\left|\chi_{B_{r}\left(x_{0}\right)}(y)\right| d y \leq \frac{\left|B_{r}\left(x_{0}\right)\right|}{\left|B_{\rho}(x)\right|}=\frac{r^{n}}{\rho^{n}}
$$

for each $x \in B_{2^{k+1}{ }_{r}}\left(x_{0}\right) \backslash B_{2^{k} r}\left(x_{0}\right)$ and each $\rho>0$. This way, the term on the lefthand side above is positive only for values $\rho>2^{k} r-r$, and the simple inequality $2^{k}-1 \geq 2^{k-1}$ which holds for all $k \geq 1$, reduces (3.17) to

$$
\frac{1}{\left|B_{\rho}(x)\right|} \int_{B_{\rho}(x)}\left|\chi_{B_{r}\left(x_{0}\right)}(y)\right| d y \leq \frac{r^{n}}{2^{n(k-1)} r^{n}}=\frac{1}{2^{n(k-1)}} .
$$

Take now the supremum in $\rho>0$ in order to get

$$
\left(\mathcal{M} \chi_{B_{r}\left(x_{0}\right)}(x)\right)^{\sigma} \leq \frac{1}{2^{\sigma n(k-1)}} .
$$


With this bound at hand, we estimate $I_{k}\left(r, x_{0}\right)$ from (3.15) as follows

$$
\begin{aligned}
I_{k}\left(r, x_{0}\right) & \leq \frac{1}{2^{\sigma n(k-1)}} \int_{B_{2^{k+1} 1_{r}}\left(x_{0}\right) \backslash B_{2^{k_{r}}}\left(x_{0}\right)}|K(x)|^{p q} d x \\
& \leq \frac{1}{2^{\sigma n(k-1)}} \int_{B_{2^{k+1} r_{r}}\left(x_{0}\right)}|K(x)|^{p q} d x \\
& \leq \frac{\left(2^{k+1} r\right)^{\gamma}}{2^{\sigma n(k-1)}} \frac{1}{\left(2^{k+1} r\right)^{\gamma}} \int_{B_{2^{k+1_{r}}\left(x_{0}\right)}|K(x)|^{p q} d x} \leq 2^{\gamma+\sigma n}\left(2^{\gamma-\sigma n}\right)^{k} r^{\gamma}\|K\|_{L^{p q, \gamma}(\Omega)}^{p q} \\
& \leq 2^{\gamma+\sigma n}\left(2^{\gamma-\sigma n}\right)^{k} r^{\gamma}\left(\|\mathbf{F}\|_{L^{p q, \gamma}\left(\Omega ; \mathbb{R}^{n}\right)}^{p q}+\|D \psi\|_{L^{p q, \gamma}\left(\Omega ; \mathbb{R}^{n}\right)}^{p q}\right)
\end{aligned}
$$

A substitution of (3.16) and (3.18) into (3.14) yields

$$
\begin{aligned}
\int_{B_{r}\left(x_{0}\right) \cap \Omega}|D u(x)|^{p q} d x & \leq c r^{\gamma}\left(\sum_{k=0}^{\infty}\left(2^{\gamma-\sigma n}\right)^{k}\right)\left(\|\mathbf{F}\|_{L^{p q, \gamma}\left(\Omega ; \mathbb{R}^{n}\right)}^{p q}+\|D \psi\|_{L^{p q, \gamma}\left(\Omega ; \mathbb{R}^{n}\right)}^{p q}\right) \\
& =c r^{\gamma}\left(\|\mathbf{F}\|_{L^{p q, \gamma}\left(\Omega ; \mathbb{R}^{n}\right)}^{p q}+\|D \psi\|_{L^{p q, \gamma}\left(\Omega ; \mathbb{R}^{n}\right)}^{p q}\right)
\end{aligned}
$$

thanks to our choice $\sigma \in(\gamma / n, 1)$ which ensures the convergence of the series above.

To complete the proof of Theorem 2.5 it remains to divide both sides above by $r^{\gamma}$ and to take the supremum with respect to $x_{0} \in \Omega$ and $r>0$ in order to get $|D u| \in L^{p q, \gamma}(\Omega)$ with the desired estimate

$$
\|D u\|_{L^{p q, \gamma}\left(\Omega ; \mathbb{R}^{n}\right)} \leq c\left(\|\mathbf{F}\|_{L^{p q, \gamma}\left(\Omega ; \mathbb{R}^{n}\right)}+\|D \psi\|_{L^{p q, \gamma}\left(\Omega ; \mathbb{R}^{n}\right)}\right) .
$$

The Proof of Corollary 2.6 is an immediate of the known pointwise properties of functions with gradients in Morrey spaces (cf. [10]) and the (A)-condition (2.1). We left the details to the reader.

\section{REFERENCES}

[1] Verena Bögelein, Frank Duzaar, and Giuseppe Mingione, Degenerate problems with irregular obstacles, J. Reine Angew. Math. 650 (2011), 107-160, DOI 10.1515/CRELLE.2011.006. MR:2770559 (2012c:35233)

[2] Verena Bögelein and Christoph Scheven, Higher integrability in parabolic obstacle problems, Forum Math. 24 (2012), no. 5, 931-972, DOI 10.1515/form.2011.091. MR2988568

[3] Sun-Sig Byun and Dian K. Palagachev, Morrey regularity of solutions to quasilinear elliptic equations over Reifenberg flat domains, Calc. Var. Partial Differential Equations 49 (2014), no. 1-2, 37-76, DOI 10.1007/s00526-012-0574-4. MR3148106

[4] S. Byun and D.K. Palagachev, Boundedness of the weak solutions to quasilinear elliptic equations with Morrey data, Indiana Univ. Math. J. 62 (2013), no. 5, 1565-1585.

[5] Sun-Sig Byun, Dian K. Palagachev, and Seungjin Ryu, Weighted $W^{1, p}$ estimates for solutions of non-linear parabolic equations over non-smooth domains, Bull. Lond. Math. Soc. 45 (2013), no. 4, 765-778, DOI 10.1112/blms/bdt011. MR3081545

[6] Sun-Sig Byun, Yumi Cho, and Lihe Wang, Calderón-Zygmund theory for nonlinear elliptic problems with irregular obstacles, J. Funct. Anal. 263 (2012), no. 10, 3117-3143, DOI 10.1016/j.jfa.2012.07.018. MR2973336

[7] Sun-Sig Byun and Lihe Wang, Parabolic equations in time dependent Reifenberg domains, Adv. Math. 212 (2007), no. 2, 797-818, DOI 10.1016/j.aim.2006.12.002. MR2329320 (2008h:35131) 
[8] Sun-Sig Byun and Lihe Wang, Nonlinear gradient estimates for elliptic equations of general type, Calc. Var. Partial Differential Equations 45 (2012), no. 3-4, 403-419, DOI 10.1007/s00526-011-0463-2. MR2984138

[9] L. A. Caffarelli, The obstacle problem revisited, J. Fourier Anal. Appl. 4 (1998), no. 4-5, 383-402, DOI 10.1007/BF02498216. MR.1658612 (2000b:49004)

[10] Sergio Campanato, Proprietà di inclusione per spazi di Morrey (Italian), Ricerche Mat. 12 (1963), 67-86. MR0156228 (27 \#6157)

[11] R. R. Coifman and R. Rochberg, Another characterization of BMO, Proc. Amer. Math. Soc. 79 (1980), no. 2, 249-254, DOI 10.2307/2043245. MR565349(81b:42067)

[12] Guy David and Tatiana Toro, Reifenberg parameterizations for sets with holes, Mem. Amer. Math. Soc. 215 (2012), no. 1012, vi+102, DOI 10.1090/S0065-9266-2011-00629-5. MR2907827

[13] Michela Eleuteri and Jens Habermann, Calderón-Zygmund type estimates for a class of obstacle problems with $p(x)$ growth, J. Math. Anal. Appl. 372 (2010), no. 1, 140-161, DOI 10.1016/j.jmaa.2010.05.072. MR2672516(2011j:49078)

[14] Lawrence C. Evans, Partial differential equations, 2nd ed., Graduate Studies in Mathematics, vol. 19, American Mathematical Society, Providence, RI, 2010. MR2597943 (2011c:35002)

[15] Avner Friedman, Variational principles and free-boundary problems, Pure and Applied Mathematics, John Wiley \& Sons Inc., New York, 1982. A Wiley-Interscience Publication. MR679313 (84e:35153)

[16] Carlos E. Kenig and Tatiana Toro, Free boundary regularity for harmonic measures and Poisson kernels, Ann. of Math. (2) 150 (1999), no. 2, 369-454, DOI 10.2307/121086. MR.1726699 (2001d:31004)

[17] David Kinderlehrer and Guido Stampacchia, An introduction to variational inequalities and their applications, Classics in Applied Mathematics, vol. 31, Society for Industrial and Applied Mathematics (SIAM), Philadelphia, PA, 2000. Reprint of the 1980 original. MR.1786735 (2002d:49001)

[18] O. A. Ladyzhenskaya and N. N. Uraltseva, Lineinye i kvazilineinye uravneniya ellipticheskogo tipa (Russian), Izdat. "Nauka", Moscow, 1973. Second edition, revised. MR0509265 $(58 \# 23009)$

[19] A. Lemenant, E. Milakis and L. V. Spinolo, On the extension property of Reifenberg-flat domains, (2012), arXiv:1209.3602.

[20] John L. Lewis and Kaj Nyström, Regularity and free boundary regularity for the p-Laplace operator in Reifenberg flat and Ahlfors regular domains, J. Amer. Math. Soc. 25 (2012), no. 3, 827-862, DOI 10.1090/S0894-0347-2011-00726-1. MR2904575

[21] Tadele Mengesha and Nguyen Cong Phuc, Weighted and regularity estimates for nonlinear equations on Reifenberg flat domains, J. Differential Equations 250 (2011), no. 5, 2485-2507, DOI 10.1016/j.jde.2010.11.009. MR2756073 (2011m:35035)

[22] Benjamin Muckenhoupt, Weighted norm inequalities for the Hardy maximal function, Trans. Amer. Math. Soc. 165 (1972), 207-226. MR0293384 (45 \#2461)

[23] Dian K. Palagachev and Lubomira G. Softova, Quasilinear divergence form parabolic equations in Reifenberg flat domains, Discrete Contin. Dyn. Syst. 31 (2011), no. 4, 1397-1410, DOI 10.3934/dcds.2011.31.1397. MR2836359(2012i:35187)

[24] Dian K. Palagachev and Lubomira G. Softova, The Calderón-Zygmund property for quasilinear divergence form equations over Reifenberg flat domains, Nonlinear Anal. 74 (2011), no. 5, 1721-1730, DOI 10.1016/j.na.2010.10.044. MR2764374 (2012c:35054)

[25] E. R. Reifenberg, Solution of the Plateau Problem for $m$-dimensional surfaces of varying topological type, Acta Math. 104 (1960), 1-92. MR0114145 (22 \#4972)

[26] José-Francisco Rodrigues, Obstacle problems in mathematical physics, North-Holland Mathematics Studies, vol. 134, North-Holland Publishing Co., Amsterdam, 1987. Notas de Matemática [Mathematical Notes], 114. MR880369 (88d:35006)

[27] C. Scheven, Existence of localizable solutions to nonlinear parabolic problems with irregular obstacles, preprint, (2011).

[28] Christoph Scheven, Gradient potential estimates in non-linear elliptic obstacle problems with measure data, J. Funct. Anal. 262 (2012), no. 6, 2777-2832, DOI 10.1016/j.jfa.2012.01.003. MR2885965 
[29] Lubomira Softova, Singular integrals and commutators in generalized Morrey spaces, Acta Math. Sin. (Engl. Ser.) 22 (2006), no. 3, 757-766, DOI 10.1007/s10114-005-0628-z. MR:2219687(2006m:42027)

[30] Alberto Torchinsky, Real-variable methods in harmonic analysis, Pure and Applied Mathematics, vol. 123, Academic Press Inc., Orlando, FL, 1986. MR869816 (88e:42001)

[31] Tatiana Toro, Doubling and flatness: geometry of measures, Notices Amer. Math. Soc. 44 (1997), no. 9, 1087-1094. MR1470167(99d:28010)

Department of Mathematical Sciences and Research Institute of Mathematics, Seoul National University, Seoul 151-747, Korea

E-mail address: byun@snu.ac.kr

Department of Mathematical Sciences, Seoul National University, Seoul 151-747, Korea

Current address: School of Mathematics, Korea Institute for Advanced Study, Seoul 120-722, Korea

E-mail address: yumicho@kias.re.kr

Dipartimento di Meccanica, Matematica e Management (DMMM), Politecnico di Bari, 70125 BARI, ITALY

E-mail address: dian.palagachev@poliba.it 